\title{
R Hegmatogenous Retinal Detachment in Cytomegalovirus Retinitis in A Post Liver Transplant-Immunocompromised Patient: Clinical and Anatomical Features and Visual Outcomes
}

\author{
Adel Alakeely*, Abdulaziz Alageely and Eman AlKahtani \\ King Khaled Eye Specialist Hospital, Saudi Arabia \\ *Corresponding Author: Adel Alakeely, King Khaled Eye Specialist Hospital, \\ Saudi Arabia.
}

DOI: $10.31080 /$ ASOP.2020.03.0097
Received: January 20, 2020

Published: February 27, 2020

(C) All rights are reserved by Adel Alakeely., et al.

\begin{abstract}
Cytomegalovirus (CMV) infection is a common viral infection in non-HIV immunocompromised posttransplant patients, which causes significant morbidity and mortality. We present a rare case of Isolated CMV retinitis in a non-HIV immunocompromised patient 7 years after a liver transplant. The 58-year-old patient was on Cell cept (mycophenolate mofetil), Tacrolimus, and Entecavir, and presented with decreased vision and floaters in the right eye. Vision in the right eye was 20/40, while examination revealed mild uveitis, retinitis, and a foveal-sparing subretinal detachment. The patient underwent a pars plana vitrectomy (PPV) with silicone oil, endolaser, and intravitreal Ganciclovir, with the silicone oil removed 10 months later. The patient's best corrected visual acuity (VA) was 20/60 in the affected eye 12 months after surgery. We speculate that his use of prophylactic Entecavir may have played a role in this unique clinical picture. We assume that the positive visual outcome was due to good VA at presentation, secondary to fovealsparing retinal detachment (RD); this entailed the use of intravitreal ganciclovir injection after surgery with systemic Ganciclovir, close follow-up, and planning surgical steps prior to the intervention.

Conclusion: Careful surgical planning with the use of intravitreal ganciclovir injection after surgery and systemic Ganciclovir may improve the visual outcome in such presentation.

Keywords: Retinal Detachment; Pars Plana Vitrectomy; Cytomegalovirus Retinitis; Liver Transplant; Immunocompromised
\end{abstract}

\section{Abbreviation}

CMV: Cytomegalovirus; VA: Visual Acuity; SB: Scleral Buckle; ValGCV: Valganciclovir; RD: Retinal Detachment;ROS: Removal Of Silicone Oil; PVR: Proliferative Vitreoretinopathy; INL: Inner Nuclear Layer; OCT: Optical Coherence Tomography; IS: Inner Segment; OS: Outer Segment

\section{Introduction}

Cytomegalovirus (CMV) infection is a common viral infection that lead to significant morbidity and mortality in non-HIV immunocompromised posttransplant patients [1]. Non-HIV related CMV retinitis has been reported in cases exposed to intensive immunosuppressive therapy for organ transplant [2]. Intraocular inflammation is more common in CMV retinitis, as seen in non-HIV immunocompromised patients than in patients with HIV infection $[3,4]$. Variable presentation of CMV retinitis in non-HIV immunocompromised patients has also been reported, with zone 1 involvement occurring in 55\% of them at presentation [5]. Retinal involvement in CMV infection typically occurs later, in comparison to other organs. A number of different mechanisms play a role in this phenomenon. First, an earlier onset with considerable damage of the retina suggests a strong virus-mediated cytopathic component, which seems to respond better to intravenous Ganciclovir. The second pattern was observed at a later point, when maintenance immunosuppression is typically lower, such that the response to intravenous Ganciclovir was less successful [6].

The low incidence of ocular CMV involvement has been linked to the prophylactic use of antiviral agents in transplants patients [5]. Nevertheless, multiple risk factors in these patients have been found to increase the risk of CMV infection. These include: CMV seropositivity before transplantation, development of CMV reactivation in the first 100 days after transplantation, presence of chronic graft-versus-host disease (GVHD), and delayed engraftment of lymphocytes [5]. Certain immunosuppressants such as Cell cept (Mycophenolate Mofetil) - which is used frequently in solid organ transplant - was found to increase the risk of complicated systemic CMV infection in organ transplant patients; this is due to impairment of both cellular and humoral immunity directed towards CMV by inhibiting both $\mathrm{T}$ and $\mathrm{B}$ lymphocyte proliferation $[7,8]$. Late CMV 
infection is commonly defined as an onset later than 3 months posttransplant, when maintenance immunosuppression is generally lower, so that valganciclovir (ValGCV) prophylaxis is discontinued. There was no association with the occurrence of rejection episodes, treatment with corticosteroids, or the early use of antiviral prophylaxis $[5,7,9]$.

Polymerase chain reaction (PCR) is an adjunct rapid analysis method used in the diagnosis and treatment of infectious retinitis in immunocompromised patients, which is both highly accurate and reproducible in the laboratory with a very low false-positive rate. Diagnostic undiluted vitreous sampling is the most appropriate diagnostic strategy; this is a function of access to greater quantities of tissue specimens for cytological analysis [10].

In term of complication, retinal detachment (RD) occurs less in non-HIV related CMV retinitis (CMVR) [11]. Despite the recent decreased incidence of CMV retinitis in general, the reported percent of RD in CMV retinitis has increased [4].Time-dependent risk and retinal surface involvement risk were studied in CMV retinitis by Freeman., et al. who found 2 elements that increase retinal detachment risk: longer duration of retinal involvement and retinal surface involvement outside the posterior pole, external to the major vascular arcades [8].

In the largest review of CMVR in HIV patients, RD occurred in $23 \%$ of cases at a median of 2 year post-infection. Patients with bilateral disease were more likely to have 1 or more retinal detachments than those with unilateral disease [12]. In non-HIV CMVR, the largest series of 13 patients revealed a similar RD incidence of $25 \%$ with a rate of $21.7 \%$ per eye per year [13].

Proliferative vitreoretinopathy (PVR) is associated with poor functional and anatomical outcomes, occurring in 29\% of cases, which is more than other types of RD [14].

Prophylaxis of RD in CMVR has been suggested, although no prospective study demonstrated a clinical significance of laser photocoagulation of the healthy retina in non-RD cases; however, the trends observed showed it to be beneficial $[2,14]$.

Management of RD in CMVR is individualized to each case. Macula-sparing RD can be treated with demarcation laser photocoagulation to the healthy retina, relieving the eye of the loss of its best corrected visual acuity (VA), temporary postoperative visual loss due to silicone-induced refractive error, and delayed visual loss due to cataracts after vitrectomy [15].
Pneumatic retinopexy can delay surgical intervention when the causative retinal break is in the superior retina, which would be in an area with no active CMV retinitis [16].

Vitrectomy with scleral buckle (SB) and silicone oil tamponade is used in almost all cases of CMV-related RD, although the choice of external and internal tamponade has been debated. In a study of 11 patients, there was an $82 \%$ success rate in reattachment with vitrectomy and silicone oil without SB [17].

Another study of 5 patients showed successful reattachment in 4 cases with vitrectomy and SB, in which gas tamponade was used instead of silicone oil [18]. Martidis., et al. combined pars plana vitrectomy (PPV) and silicone oil infusion with simultaneous placement of a Ganciclovir implant, which showed anatomical success in all 10 cases with good control of retinitis [18].

Redetachment happens more frequently in CMV-related RD, reaching up to $30 \%$ in some studies [19]. Morrison., et al. reported redetachment in 53\% after removal of silicone oil (ROS), which occurred 4 months after the procedure was performed. Strikingly, redetachment after ROS occurred in most cases when combined with cataract surgery, although SB did not make any difference in redetachment [12].

This trend has shifted in recent studies, in which reported anatomical success after ROS was 82\% [20]. Another study showed better anatomical results with $23 \mathrm{~g}$ compared to $20 \mathrm{~g}$ vitrectomy, but this was not statistically significant [20].

Final visual outcome was poor. However, many issues play a role in poor vision: these include persistent detachment or redetachment, optic atrophy, epiretinal membrane, corneal scar, vitritis and macular scarring. Other potentially treatable problems include cataracts, refractive errors from silicone oil, and SB. Final VA was strongly correlated with VA at the time of RD diagnosis, given the duration between the diagnosis and surgery [11,20,21]. We report a non-HIV immunocompromised patient who presented 7 years after liver transplant with retinal detachment secondary to CMV retinitis.

\section{Case Report}

A 58-year-old male with a history of a liver transplant 7 years earlier due to chronic viral hepatitis B with regular follow-up; he was receiving Cell cept, Tacrolimus (FK 506), and Entecavir. He presented with a history of decreased vision and floaters in his right eye, which started a month earlier and increased over the past week. 
R Hegmatogenous Retinal Detachment in Cytomegalovirus Retinitis in A Post Liver Transplant-Immunocompromised Patient: Clinical and Anatomical Features and Visual Outcomes

His VA was 20/40 in the right eye, and 20/25 in the left eye with normal intraocular pressure.

Examination of the right eye revealed occasional anterior chamber cells, mild vitritis with sparse vitreous cells, and multifocal white vitreous exudates. A retinal exam showed a few spots of retinal hemorrhage, small multifocal retinal vasculitis, periphlebitis, and a shower of white retinitis lesions with a brushfire-like border associated with a secondary large atrophic nasal break of the disc. A foveal-sparing subtotal retinal detachment was seen following the nasal retinal break (Figure 1). A schisis-like picture was evident at the inner nuclear layer of the macula. The high-resolution images via optical coherence tomography (OCT) (Figure 2) allowed precise and direct localization of retinoschisis. A B-scan ultrasound showed the foveal-sparing RD.

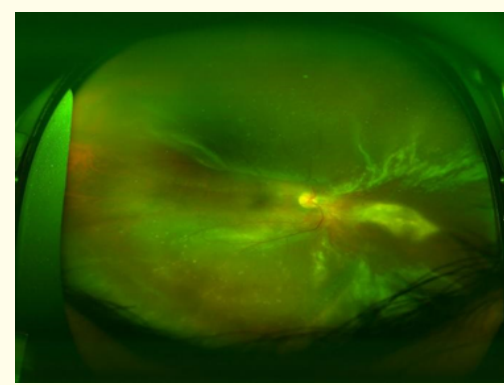

Figure 1: Fundus photo showing foveal sparing subtotal retinal detachment of the affected eye.

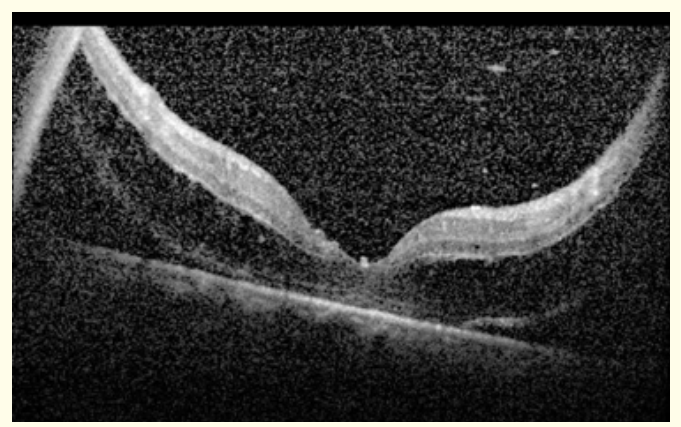

Figure 2: OCT showing schisis like picture.

The patient underwent PPV with an endolaser and intravitreal Ganciclovir $2 \mathrm{mg} / 0.1 \mathrm{~mL}$ and silicone oil 5000 centistokes injections, with appropriate surgical management. PCR testing for a rapid analysis of the vitreous sample was positive for CMV. The patient was seen by his hepatologist, who started him on Valcyte (valganciclovir hydrochloride) $450 \mathrm{mg}$ for 6 months.

On his first follow-up, his VA was $2 / 200$ in the right eye, while examination showed marked retinal hemorrhages with no new infiltrates or anterior chamber reaction. The retinal hemorrhages resolved on subsequent follow-up visits and the patient underwent cataract surgery with posterior chamber intraocular lens (IOL) implantation without silicone oil removal. After finishing the course of systemic antiviral drugs, the patient remained stable with a flat retina with the use of silicone oil. The patient safely underwent oil removal 10 months after his primary surgery with excellent anatomical and functional success, with his best corrected vision 12 months after primary surgery, along with $20 / 60$ in the right eye (Figure 3 and 4 ).

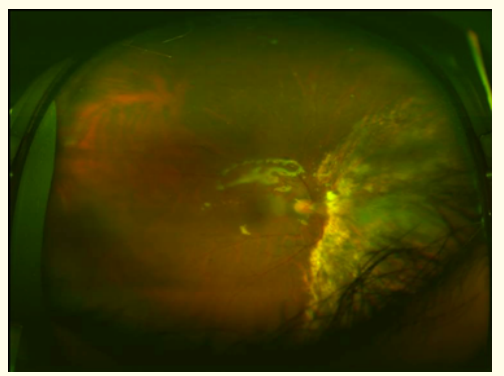

Figure 3: Fundus photo showing retinal reattachment after silicone oil removal.

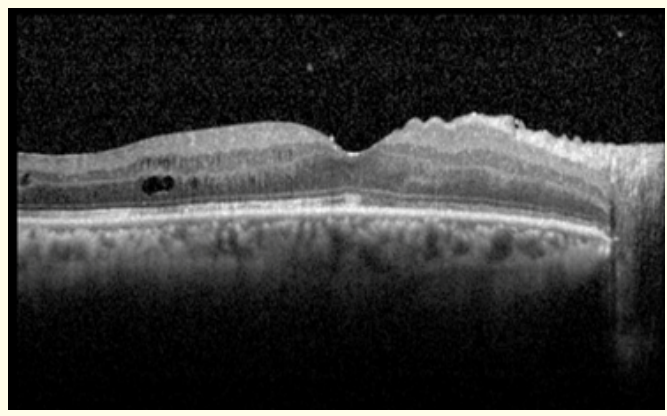

Figure 4: OCT showing the fovea after silicone oil removal.

\section{Discussion}

This is a rare case of isolated CMV infection in a non-HIV immunocompromised patient post-liver transplant. It demonstrates an unusual infection with mild posterior inflammation without retinal hemorrhages, in contrast to other transplant patients who show more aggressive peripheral inflammation with marked retinal hemorrhages [4]. This inflammation was evident on follow-up visits after initiating a more aggressive antiviral regimen. The presenting retinal detachment was caused by a posterior lesion which typically carries a lower risk than that of a peripheral one [9]. Other factors that may have contributed to CMV retinitis include the use of Cellcept and Tacrolimus, which have been reported in cases with this infection $[13,22]$. Anti-Hepatitis B virus agents such as Enteca- 
vir were reported to have anti-CMV activity [8]. We speculate that this patient's use of prophylactic Entecavir may have contributed to this distinctive clinical picture [14]. Most reported cases occur within a year of transplant. The late onset of the infection might necessitate a longer follow-up to screen for this problem [2].

Effects of retinal necrosis have been demonstrated by optical coherence tomography (OCT), with extensive necrosis of the fovea, resulting in significant destruction of the retinal nerve fiber layer and formation of multiple cystic spaces connected with loss of the inner segment (IS)/outer segment (OS) junction of the photoreceptors [23].

We reported changes seen with spectral domain OCT that - to the best of our knowledge - were not noted before, in which thickening in retinal layers was observed more prominently in the outer layers, along with foveal-sparing retinal detachment and a foveomacular schisis picture with the split at the inner nuclear layer (INL), thereby separating the retina into 2 layers. Vitreous condensations were also demonstrated with OCT.

The surgical reattachment of the retina was successful, as the repair technique was crucial to maintaining the success of the first procedure. Vitrectomy with silicone oil as the initial surgical procedure was the most effective approach. Successful anatomic reattachment of the retina was achieved in $70 \%$ of patients having this procedure [24]. We elected not to remove silicone oil when the patient underwent cataract surgery with IOL implantation: this was becauses of a possible risk of redetachment when done simultaneously with cataract extraction [12].

We assume that the positive visual outcome was due to good $\mathrm{VA}$ at presentation, secondary to foveal-sparing $\mathrm{RD}$, using the intravitreal Ganciclovir injection at the end of surgery, in addition to systemic Ganciclovir, close follow-up, and planning surgical steps prior to intervention.

\section{Conclusion}

Aware of atypical presentation of CMV infection in a non-HIV immunocompromised patient post-liver transplant is critical in making prompt diagnosis and a careful surgical planning with the use of intravitreal ganciclovir injection after surgery and systemic Ganciclovir may improve the visual outcome in such presentation. It is clear that one case report is insufficient to warrant general recommendations; however, we believe our case reflects careful surgical planning that might be useful to others.

\section{Acknowledgements}

I would like to express my thanks and appreciation to Dr Adel Alakeely on his generous help in this study

\section{Financial Support}

None. No conflicting relationship exists for any author.

\section{Declarations}

\section{Availability of Data and Material}

Not applicable to this article as no datasets were generated or analysed during the current study.

\section{Competing Interests}

Not applicable.

\section{Funding}

No financial interest in this study.

\section{Authors' Contributions}

Not applicable

\section{Bibliography}

1. K Mwintshi and DC Brennan. "Prevention and management of cytomegalovirus infection in solid-organ transplantation". EXpert Review Anti-Infectious Therapy 5.2 (2007): 295-304.

2. A J Eid., et al. "Clinical features and outcome of cytomegalovirus retinitis after transplantation". Transplant Infectious Disease 10.1 (2008): 13-18.

3. WR Freeman., et al. "Risk factors for development of rhegmatogenous retinal detachment in patients with cytomegalovirus retinitis". American Journal Ophthalmology 116.6 (1993): 713-20.

4. R Singh., et al. "Outcome of surgery in post-cytomegalovirus retinal detachment: experience before and in the era of highly active anti-retroviral therapy in Indian eyes". Indian Journal Ophthalmology 61. 11 (2013): 636-639.

5. L Lu., et al. "Long-term follow-up of cytomegalovirus retinitis in non-HIV immunocompromised patients: clinical features and visual prognosis". American Journal of Ophthalmology 165.1 (2016): 145-153.

6. A Egli., et al. "Cytomegalovirus-associated chorioretinitis after liver transplantation: case report and review of the literature". Transplantation Infectious Disease 10.1 (2008): 27-43.

7. L Hambach., et al. "Increased risk of complicated CMV infection with the use of mycophenolate mofetil in allogeneic stem cell transplantation". Bone Marrow Transplantation 29.1 (2002): 903-906. 
8. K Pathanapitoon., et al. "Clinical manifestations of cytomegalovirus-associated posterior uveitis and panuveitis in patients without human immunodeficiency virus infection". JAMA Ophthalmology 131.5 (2013): 638-645.

9. O Shibolet., et al. "Late cytomegalovirus disease following liver transplantation”. Transplantation International 16.11 (2003): 861-865.

10. T Harper., et al. "Polymerase Chain Reaction Analysis of Aqueous and Vitreous Specimens in the Diagnosis of Posterior Segment Infectious Uveitis". American Journal Ophthalmology 147.1 (2009): 140-147.

11. MB Mowa., et al. "Inhibition of hepatitis B virus replication by helper dependent adenoviral vectors expressing artificial anti-HBV pri-miRs from a liver-specific promoter". Biomedical Research International 2014 (2014): 718-743.

12. VL Morrison., et al. "Results of silicone oil removal in patients with cytomegalovirus retinitis related retinal detachments". American Journal of Ophthalmology 140.5 (2005): 786-793.

13. JJ Lee., et al. "Occurrence and reactivation of cytomegalovirus retinitis in systemic lupus erythematosus with normal CD4(+) counts". Eye 20.5 (2006): 618-621.

14. J Cukuranovic., et al. "Viral infection in renal transplant recipients". Scientific World Journal (2012).

15. TR Vrabec. "Laser photocoagulation repair of macula-sparing cytomegalovirus-related retinal detachment". Ophthalmology 104.12 (1997): 2062-2067.

16. PF McAuliffe and MH Heinemann. "The use of pneumatic retinopexy to delay surgical repair of a retinal detachment associated with the ganciclovir intraocular device". Ophthalmic Surgery Lasers 29.3 (1998): 244-246.

17. JE Nasemann., et al. "Early pars plana vitrectomy without buckling procedure in cytomegalovirus retinitis-induced retinal detachment". Retina 15.2 (1995): 111-116.

18. A Martidis., et al. "Treating cytomegalovirus retinitis-related retinal detachment by combining silicone oil tamponade and ganciclovir implant". Ophthalmic Surgery Lasers 33.2 (2002): 135-139.

19. G Mathur., et al. "Clinical Outcomes of Retinal Detachment Surgery following Cytomegalovirus Retinitis in Patients on Highly Active Anti-retroviral Therapy for Acquired Immune Deficiency Syndrome". Ocular Immunology Inflammation and Infection 23.5 (2015): 400-404.
20. VP Dave., et al. "Results of silicone oil removal in post-cytomegalovirus retinitis-related retinal detachment". Journal Ophthalmic Inflammation and Infection 2.3 (2012): 153-155.

21. SK Gore., et al. "Cytomegaloviral retinitis-related retinal detachment: outcomes following vitrectomy in the developing world". International Ophthalmology 34.2 (2014): 205-210.

22. AP Ciardella., et al. "Cytomegalovirus retinitis and FK 506". American Journal Ophthalmology 136.2 (2003): 386-389.

23. LL Sun., et al. "Optical coherence tomography changes in macular CMV retinitis". Digital Journal Ophthalmology 18.2 (2012): 1-4.

24. DA Jabs., et al. "Retinal detachments in patients with cytomegalovirus retinitis". Archives Ophthalmology 109.1 (1991): 794-799.

\section{Assets from publication with us}

- Prompt Acknowledgement after receiving the article

- Thorough Double blinded peer review

- Rapid Publication

- Issue of Publication Certificate

- High visibility of your Published work

Website: www.actascientific.com/

Submit Article: www.actascientific.com/submission.php

Email us: editor@actascientific.com

Contact us: +919182824667 